\title{
EM Wave Transport 2D and 3D Investigations
}

\author{
Rajveer S Yaduvanshi \\ ECE Deparment \\ AIT, Govt of Delhi, India-110031 \\ email:yaduvanshirs@yahoo.co.in
}

\author{
Harish Parthasarathy \\ ECE Department \\ NSIT, Govt of Delhi, India-110075 \\ email-harishp@nsit.ac in
}

\begin{abstract}
Boltzmann Transport Equation [1-2] has been modelled in close conjunction with Maxwell's equation, investigations for 2D and 3D transport carriers have been proposed. Exact solution of Boltzmann Equation still remains the core field of research. We have worked towards evaluation of 2D and 3D solutions of BTE. Application of our work can be extended to study the electromagnetic wave transport in upper atmosphere i.e. ionosphere. We have given theoretical and numerical analysis of probability density function, collision integral under various initial and final conditions. Modelling of coupled Boltzmann Maxwell's equation taking binary collision and multi species collision terms has been evaluated. Solutions of Electric Field (E) and Magnetic Field (B) under coupled conditions have been obtained. PDF convergences under the absence of electric field have been sketched, with an iterative approach and are shown in figure 1. Also 3D general algorithm for solution of BTE has been suggested.
\end{abstract}

Keywords- Boltzmann Transport Equation, Probability Distribution Function, Coupled BTE-Maxwell's.

\section{INTRODUCTION}

BTE is an integral differential equation used for characterizing carrier transport in semiconductor [5-6] and gases distribution in space $f(x, v, t)$. The Boltzmann equation applies to a quantity known as the distribution function, which describes this non-equilibrium state mathematically [1] and specifies how quickly and in what manner the state of the gas changes when the disturbing forces are varied. BTE shall compute average behaviour of the system in terms of distribution function of time. Evolution of distribution function is governed by Boltzmann Transport Equation. Boltzmann Transport Equation can be solved by mathematical and numerical techniques. Here distribution function can be a function of seven variables i.e. three physical space, three volume space and one time. It shall provide complete description of the state of gas. The distribution function carries information about the positions and velocities of the particles at any time. There can be two broad methods to solve the BTE. The first method consists in directly discretizing and solving the BTE using standard numerical methods [1-2] for differential equation. The second, called the Monte Carlo (MC) method, solves the BTE as being the stationary solution of a stochastic differential equation [3-4]. BTE structure due to high dimensionality is hard to solve.

One could describe a gas flow in classical physics by giving position and velocity of all molecules at any instant of time. It means information about flow of gas, electron and ions can be known by solving BTE with proper initial conditions and boundary conditions. The solution of BTE is PDF (3-4), which is a function of position and velocity of particles and the time variables. Distribution Function shall be derived by concentration of kinetic energy and moments due to applied force. Distribution function can be also characterized as current density.

A statistical approach [5-7] can be taken instead of defining position and velocity of each molecule. Using the construct of ensemble, a large number of independent systems evolving independently but under same dynamics, can be characterized by density function, which gives the probability that an ensemble member can be found in some elemental volume in phase space, which has been very well explained in reference [8-9].

In a state of equilibrium a gas of particles has uniform composition and constant temperature and density. If the gas is subjected to a temperature difference [8] or disturbed by externally applied electric, magnetic, or mechanical forces, it will be set in motion and the temperature, density, and composition may become functions of position and time, in other words, the gas moves out of equilibrium and specifies how quickly and in what manner state changes when disturbing forces are controlled. Equilibrium can be disturbed by temperature change, external force, magnetic force and mechanical force.

Here we have proposed three different models i.e. when BTE is at equilibrium state, second when force is applied but no field presents and third condition is when both force and field exists simultaneously. We have developed BTE formulations for two dimensional and three dimensional solutions. We have studied transport parameters i.e. charge density, current density, magnetic potential and electric potential, electric field and magnetic field. General purpose algorithms for 3D analysis have also been developed. This developed modeling theory can be very useful for tolerance analysis in chip designing in microelectronics. We have solved coupled equations by finite difference numerical method.

We have organized our paper in five sections. Section 1 gives us the concept of BTE. Section 2 describes BTE formulations [10]. Section 3 presents modeling and simulations of BTE. Section 4 deals with multi species collision Section 5 
presents coupled modeling of EM transport. Section 6 concludes the paper.

$$
\begin{aligned}
& \text { Abbreviations used in the text } \\
& \text { v=velocity of particles } \\
& \mathrm{f}=\text { force on particles } \\
& \mathrm{m}=\text { mass of particle } \\
& \mathrm{x}=\text { displacement } \\
& \text { BTE=Boltzmann Transport equation } \\
& \text { PDF= Probability Density Function } \\
& \text { EM= Electromagnetic wave. }
\end{aligned}
$$

\section{BTE FORMULATIONS}

Here distribution function can be a function of seven variables for 3D model i.e. three physical space, three volume space and one time. It shall provide complete description of the state of gas. There can be two broad methods to solve the BTE

Standard numerical methods for differential equation.

The Monte Carlo (MC) method solves the BTE as being the stationary solution of a stochastic differential equation.

Here we shall work for numerical solution for all investigations.

$\underline{\text { BTE }}$ under Steady State Conditions

$$
\frac{\partial f}{\partial t}+v \frac{\partial f}{\partial x}+\frac{F}{m} \frac{\partial f}{\partial v}=0
$$

BTE under Equilibrium conditions [2]

$$
\mathbf{f}(\overrightarrow{\mathbf{x}}, \overrightarrow{\mathbf{v}}, \mathbf{t})\left(\frac{\partial}{\partial \mathrm{t}}+\overrightarrow{\mathbf{v}} \nabla \mathbf{x}+\frac{\mathrm{F}}{\mathbf{m}} \Delta \mathbf{v}\right)=\mathbf{0}
$$

$2 \mathrm{D}$ representation of BTE

$$
\frac{\delta f}{\delta x}(\boldsymbol{x}, \boldsymbol{V} \boldsymbol{x})=-V \boldsymbol{x} \frac{\delta f\left(x_{i} V \boldsymbol{x}\right)}{\delta x}
$$

3 D representation of BTE under RTA

$$
\frac{\partial \mathbf{f}}{\partial \mathrm{t}}+\mathbf{v} \frac{\partial \mathrm{f}}{\partial x}+\frac{\mathrm{F}}{\mathrm{m}} \frac{\partial \mathrm{f}}{\partial \mathrm{v}}=-\frac{f-f c}{\tau}
$$

BTE when collision term is accounted for

$$
\frac{\partial f}{\partial t}+v \frac{\partial f}{\partial x}+\frac{F}{m} \frac{\partial f}{\partial v}=\left(\frac{\partial f}{\partial t}\right) \operatorname{coll}
$$

We have discretized the above $2 \mathrm{~d}$ and 3 differential equations developed and subjected for numerical solution. The output is the PDF w. $r$.t position and velocity at different times. The PDF is normalized, where intensity of the image represents its relative value. Solution of BTE under constant EM field and force applied is computed as presented in fig1, it is assumed that there is no collision and initial function is Gaussian in nature. Given below are the 2Dand 3D computed transport models.

$$
\begin{aligned}
& \frac{d f}{d x}(x, \mathbf{V x}, t)+\mathbf{V} \mathbf{x} \frac{d f}{d x}+\frac{f x}{m} \frac{d f}{d V \mathbf{x}}=0 \\
& \frac{d f}{d x}(x, V \mathbf{x}, t)+\mathbf{V} \mathbf{x} \frac{d f}{d x}+\frac{e \cdot E o}{m} \frac{d f}{d V \mathbf{x}}=0
\end{aligned}
$$

Now for six space dimensions seven variables are considered. The distribution function $\mathrm{f}(\mathrm{x}, \mathrm{v}, \mathrm{t})$ for $3 \mathrm{D}$ represents $\mathrm{x}$ as position vector and $\mathrm{v}$ as velocity vector.

$$
\begin{aligned}
& \frac{d f}{d t}(x, y, z, V x, V y, V z, t) \\
& +V x \frac{d f}{d x}+V y \frac{d f}{d y}+V z \frac{d f}{d z} \\
& +\frac{e}{m}(E x+V y B z-V z B y) \frac{d f}{d V x} \\
& \quad+\frac{e}{m}(E y+V x B x-V x B z) \frac{d f}{d V y} \\
& \quad+\frac{e}{m}(E z+V x B y-V y B x) \frac{d f}{d V z} \\
& =0
\end{aligned}
$$

Here we have to find $\frac{\mathrm{df}}{\mathrm{dt}}, \frac{\mathrm{df}}{\mathrm{dx}}$ and $\frac{\mathrm{df}}{\mathrm{dv}}$. Here of intensity of image represents normalized magnitude of PDF. These plots have been obtained at different time and convergence at equilibrium is presented with initial field as Gaussian.

\section{A. $2 D$ PLOTS}
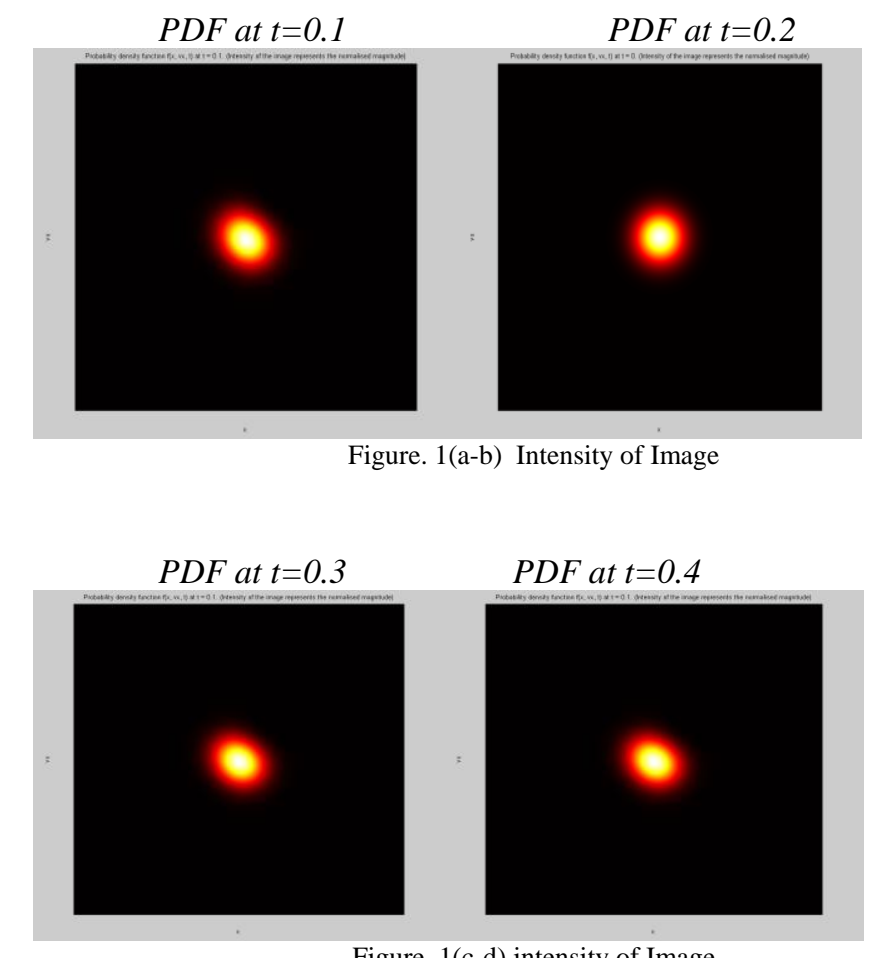

Figure. 1(c-d) intensity of Image

$P D F$ at $t=0.7$

$P D F$ at $t=0.6$ 
(IJACSA) International Journal of Advanced Computer Science and Applications,
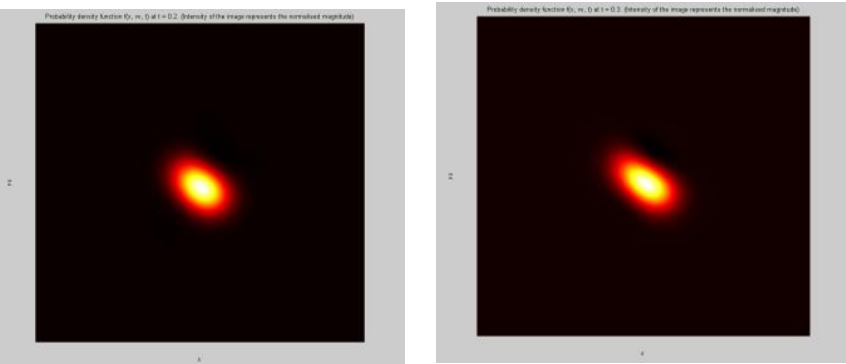

Figure.1 (e-f) Intensity of Image

PDF at $t=0.7$

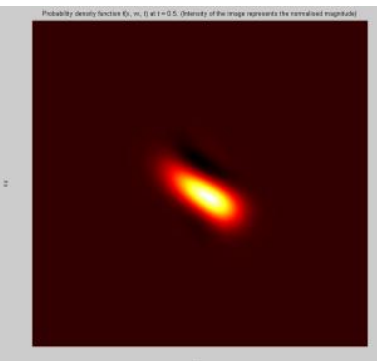

Figure. 1(g-h) Intensity of Image

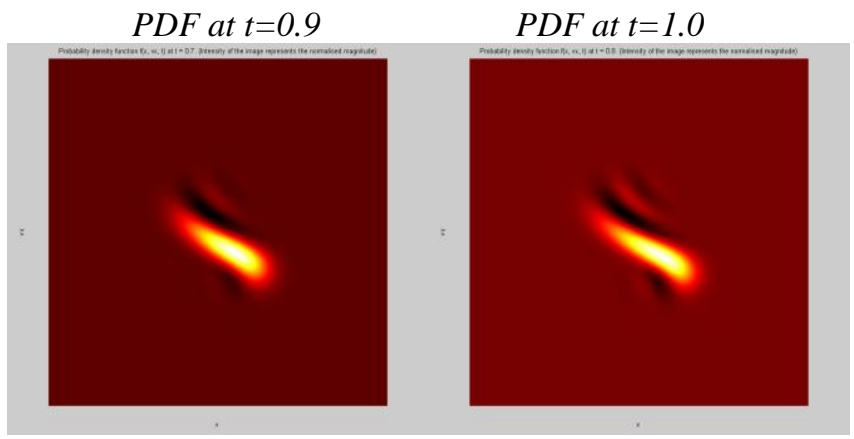

Figure.1 (i-J) Intensity of Image

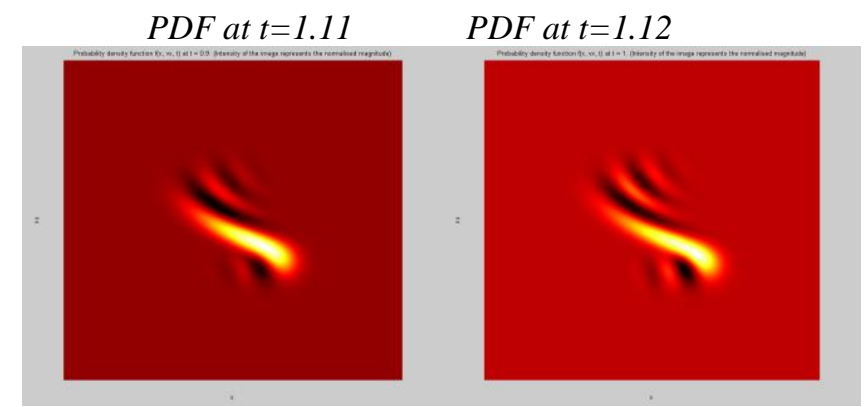

Figure. 1 (1-m) Intensity of Image
B. $3 D$ PLOTS

Vol. 1, No. 6, December 2010

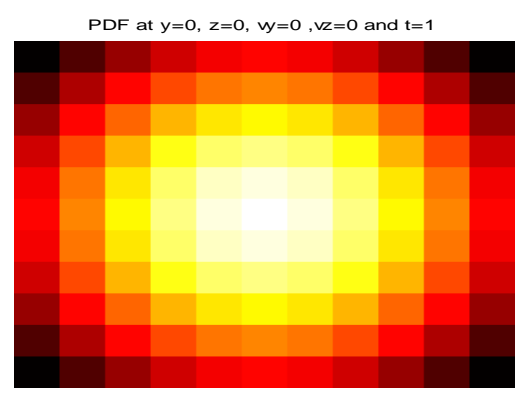

Figure. 2(a) Intensity of Image

PDF at $x=0, z=0, v x=0, v z=0$ and $t=1$

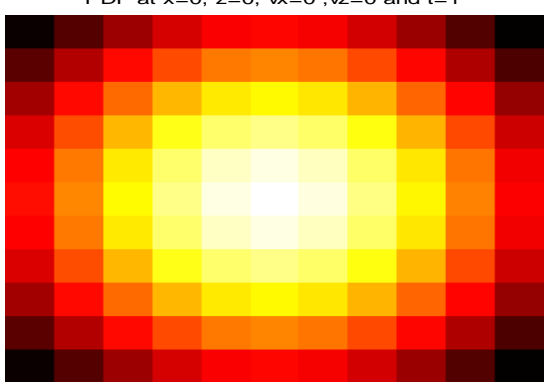

Figure 2(b) Intensity of Image

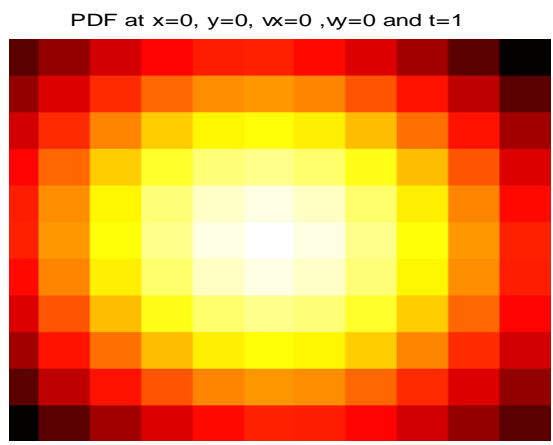

Figure 2(c) Intensity of Image

PDF at $y=0, z=0, v y=0, v z=0$ and $t=2$

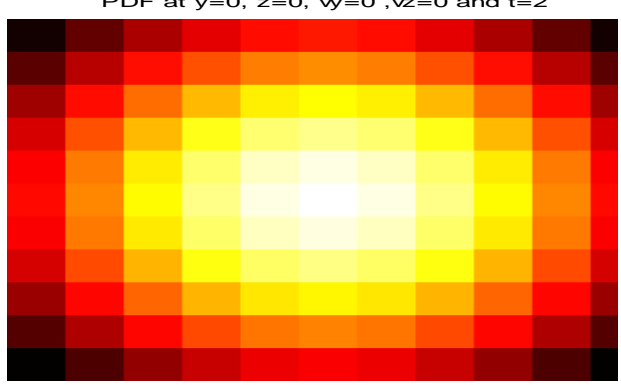

Figure 2(d) Intensity of Image 


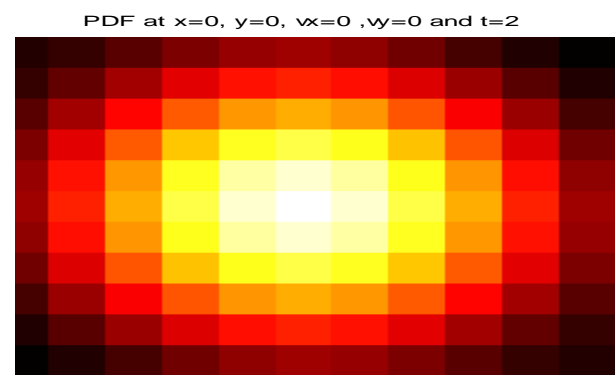

Figure 2(e) Intensity of Image

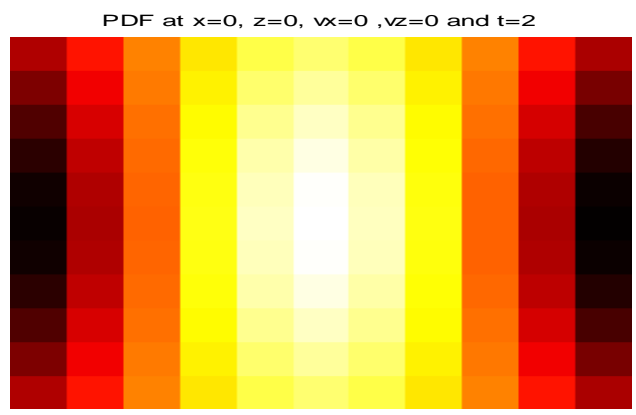

Figure 2(f) Intensity of Image

The ensemble is characterized by density function which gives probability that an ensemble member can be found in some elemental volume in phase space.

We have assumed two particles collision term. Equations (7-8) presents collision terms. Here

$$
\begin{aligned}
& \frac{d f}{d t}+v \frac{d f}{d x}+\frac{f}{m} \frac{d f}{d v}= \\
& \iint v \int \Omega\left(f^{\prime} f^{\prime} 1 f_{-} f(f 1) v \sigma d^{2} \Omega d^{3} v\right.
\end{aligned}
$$$$
\text { (7) }
$$

Where

$\mathrm{t}=$ time

$\mathrm{v}=$ molecular velocity,

$\mathrm{v} 1=$ Pre Collision

$\mathrm{V}^{\prime}=$ post collision velocity

V'1=post collision velocity

$\mathrm{F}=$ external force

$\mathrm{M}=$ mass of the molecule

$$
\mathrm{f} 1=\mathrm{f}(\mathrm{x}, \mathrm{v} 1, \mathrm{t})
$$

$\mathrm{f}^{\prime}=\mathrm{f}(\mathrm{x}, \mathrm{v} 1, \mathrm{t})$ here prime values represents post collision conditions due to conservation

$$
\begin{aligned}
& \mathrm{V}=\mathrm{v}-\mathrm{v} 1 \\
& \mathrm{~V}=|\mathrm{V}|=\left|\mathrm{V}^{\prime}\right|
\end{aligned}
$$

$\Omega$ Solid angle is deflection angle of relative velocity. $\sigma$ collision cross section will depend on potential, scattering and velocity of particles. Collision cross section is defined by probability that a collision between two molecules will result in a given pair of post collision velocities. The integration over velocity $\mathrm{v} 1$ from $-\infty$ to $\infty$ in all dimensions while integrating over $\Omega$ extends over unit sphere.

$$
\begin{aligned}
& \left(\frac{\partial f}{\partial t}\right) \operatorname{coll}= \\
& \int v 1 \int \Omega\left(f^{\prime} f^{\prime} 1 \ldots f f 1\right) v \sigma d^{2} \Omega d^{3} v
\end{aligned}
$$

Collision integral is five dimensional integral that must be evaluated for every point in physical space, every point in time and every point in velocity space. Collision cross section $\sigma$ will depend on intermolecular potential, pre and post collision velocities and scattering angle $\Omega$ [9-11] , though it is constant for hard sphere molecules. $\sigma$ direction describes the probability density to a certain change of velocity. Collision integral evolution approach can be directly applied to solve high dimensional problems. The term $\int \mathrm{v} 1 \int \Omega\left(\mathrm{f}^{\prime} \mathrm{f}_{1}{ }_{1} \mathrm{ff}_{1}\right) \mathrm{v}$ $\sigma \mathrm{d}^{2} \Omega \mathrm{d}^{3} \mathrm{v}$ represents net rate at which molecular enter the point of interest in phase space due to collision and $\int \mathrm{v} 1 \int \Omega$ $\left(f^{\prime} f_{1}^{\prime}{ }_{1}-f f_{1}\right) \vee \sigma d^{2} \Omega d^{3} v$ represents the net rate at which molecules are scattered out. Both terms are integrated over all possible pre collision velocity and all possible collision angles. We have also developed general purpose 3D algorithm for implementation as mentioned below:

\section{BTE 3DGeneral Purpose Algorithm:}

Clear all;

Clc;

Close all;

$\%$ for a variable space of $101^{\wedge} 6$, it might take hours to compute the result as it may require large amount of memory.

len $=101$;

Centre $=$ round $(($ len +1$) / 2)$;

$\mathrm{f}=$ zeros (len, len, len, len, len, len);

Stdev = 5;

$\%$ initializes the prior PDF as a Gaussian distribution

$\%$ that the function can be differentiated properly, and yet not extend to.

$\%$ the ends of variable space. For a variable size of len, even larger.

$\%$ values can be considered.

For $\mathrm{x}=1$ : len

For $\mathrm{y}=1$ : len

For $\mathrm{z}=1$ : len

For $\mathrm{vx}=1$ : len

For vy $=1$ : len

For $\mathrm{vz}=1:$ len

$\mathrm{F}(\mathrm{x}, \mathrm{y}, \mathrm{z}, \mathrm{vx}, \mathrm{vy}, \mathrm{vz})=$

$\exp \left(-\left((\mathrm{x}-\text { centre })^{\wedge} 2+(\mathrm{y}-\text { centre })^{\wedge} 2+(\mathrm{z}-\text { centre })^{\wedge} 2+(\mathrm{vx}-\right.\right.$

centre $\left.\left.)^{\wedge} 2+(\text { vy-centre })^{\wedge} 2+(\text { vz-entre })^{\wedge} 2\right) /\left(2^{*} \operatorname{stdev}^{\wedge} 2\right)\right)$;

End

End 


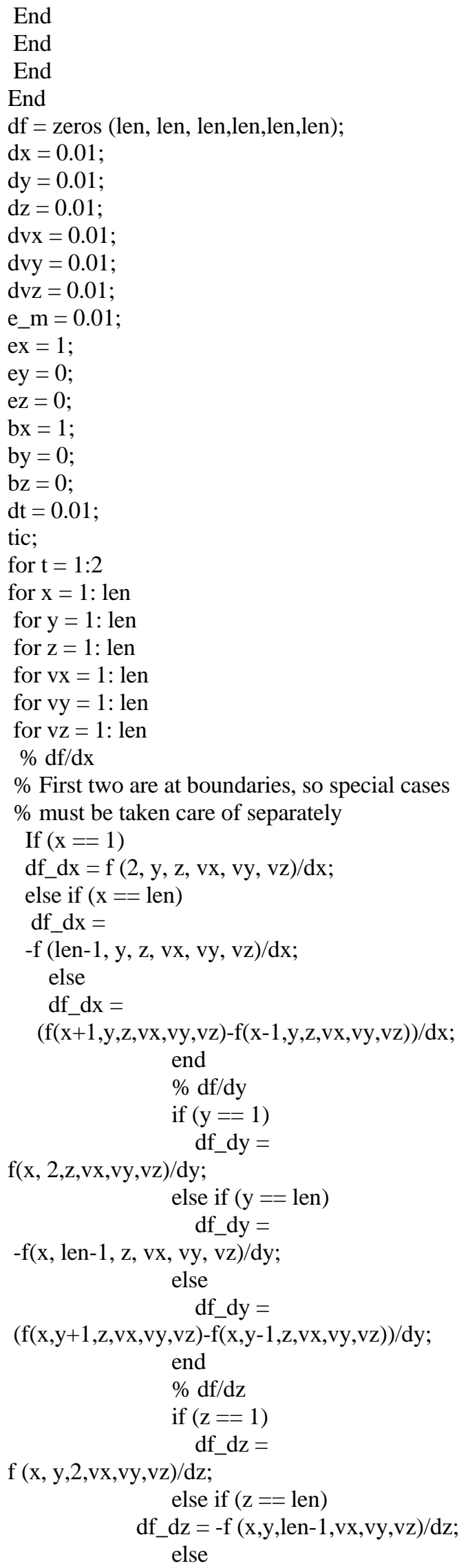

End

End

End

End

df = zeros (len, len, len,len,len,len);

$\mathrm{dx}=0.01$;

$\mathrm{dy}=0.01$

$\mathrm{dz}=0.01$

$\operatorname{dvx}=0.01$;

$\mathrm{dvy}=0.01$;

$\mathrm{dvz}=0.01$

e_m $=0.01$;

ex $=1$;

ey $=0$;

$\mathrm{ez}=0$;

$\mathrm{bx}=1$;

by $=0$;

$\mathrm{bz}=0$;

$\mathrm{dt}=0.01$

tic;

for $\mathrm{t}=1: 2$

for $\mathrm{x}=1$ : len

for $y=1$ : len

for $\mathrm{z}=1$ : len

for $\mathrm{vx}=1$ : len

for $\mathrm{vy}=1$ : len

for $\mathrm{vz}=1:$ len

$\% \mathrm{df} / \mathrm{dx}$

$\%$ First two are at boundaries, so special cases

$\%$ must be taken care of separately

If $(x==1)$

df_dx $=f(2, y, z, v x, v y, v z) / d x$;

else if $(x==$ len $)$

df_dx =

-f (len-1, y, z, vx, vy, vz)/dx;

else

df_dx $=$

$(\mathrm{f}(\mathrm{x}+1, \mathrm{y}, \mathrm{z}, \mathrm{vx}, \mathrm{vy}, \mathrm{vz})-\mathrm{f}(\mathrm{x}-1, \mathrm{y}, \mathrm{z}, \mathrm{vx}, \mathrm{vy}, \mathrm{vz})) / \mathrm{dx}$;

end

$\% \mathrm{df} / \mathrm{dy}$

if $(\mathrm{y}==1)$

df_dy =

$\mathrm{f}(\mathrm{x}, 2, \mathrm{z}, \mathrm{vx}, \mathrm{vy}, \mathrm{vz}) / \mathrm{dy}$;

else if $(y==$ len $)$

$$
\text { df_dy = }
$$

-f(x, len-1, z, vx, vy, vz)/dy;

else

df_dy $=$

$(\mathrm{f}(\mathrm{x}, \mathrm{y}+1, \mathrm{z}, \mathrm{vx}, \mathrm{vy}, \mathrm{vz})-\mathrm{f}(\mathrm{x}, \mathrm{y}-1, \mathrm{z}, \mathrm{vx}, \mathrm{vy}, \mathrm{vz})) / \mathrm{dy}$;

end

$\% \mathrm{df} / \mathrm{dz}$

if $(\mathrm{z}==1)$

$\mathrm{f}(\mathrm{x}, \mathrm{y}, 2, \mathrm{vx}, \mathrm{vy}, \mathrm{vz}) / \mathrm{dz}$;

$$
\text { df_dz }=
$$

else if $(z==$ len $)$

df_dz = -f $(x, y, l e n-1, v x, v y, v z) / d z$;

else

Vol. 1, No. 6, December 2010

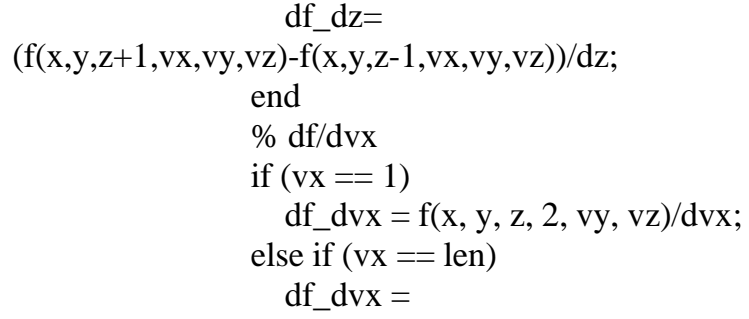

df_dz=

$-f(x, y, z$, len-1, vy, vz)/dvx;

else

df_dvx $=$

$(\mathrm{f}(\mathrm{x}, \mathrm{y}, \mathrm{z}, \mathrm{vx}+1, \mathrm{vy}, \mathrm{vz})-\mathrm{f}(\mathrm{x}, \mathrm{y}, \mathrm{z}, \mathrm{vx}-1, \mathrm{vy}, \mathrm{vz})) / \mathrm{dvx}$;

end

$\% \mathrm{df} / \mathrm{dvy}$

if $(\mathrm{vy}==1)$

df_dvy $=f(x, y, z, v x, 2, v z) / d v y$;

else if $(v y==$ len $)$

df_dvy =

$-\mathrm{f}(\mathrm{x}, \mathrm{y}, \mathrm{z}, \mathrm{vx}$, len-1, vz)/dvy;

else

df_dvy =

$(\mathrm{f}(\mathrm{x}, \mathrm{y}, \mathrm{z}, \mathrm{vx}, \mathrm{vy}+1, \mathrm{vz})-\mathrm{f}(\mathrm{x}, \mathrm{y}, \mathrm{z}, \mathrm{vx}, \mathrm{vy}-1, \mathrm{vz})) / \mathrm{dvy}$;

end

$\% \mathrm{df} / \mathrm{dvz}$

if $(\mathrm{vZ}==1)$

df dvz $=$

$\mathrm{f}(\mathrm{x}, \mathrm{y}, \mathrm{z}, \mathrm{vx}, \mathrm{vy}, 2) / \mathrm{dvz}$;

else if $(\mathrm{vz}==$ len $)$

df $d v z=$

$-\mathrm{f}(\mathrm{x}, \mathrm{y}, \mathrm{z}, \mathrm{vx}, \mathrm{vy}$, len-1)/dvz;

else

df $d v z=$

$(\mathrm{f}(\mathrm{x}, \mathrm{y}, \mathrm{z}, \mathrm{vx}, \mathrm{vy}, \mathrm{vz}+1)-\mathrm{f}(\mathrm{x}, \mathrm{y}, \mathrm{z}, \mathrm{vx}, \mathrm{vy}, \mathrm{vz}-1)) / \mathrm{dvz}$;

end

$\% \mathrm{df}=$

$(v x d f / d x+v y d f / d y+v z d f / d z+$

$(e x+v y b z-v z$ by $) d f / d v x+(e y+v x b z-v z b x)$

$\mathrm{df} / \mathrm{dvy}+(\mathrm{ez}+\mathrm{vx}$ by $-\mathrm{vy}$ bx $) \mathrm{df} / \mathrm{dvz}) * \mathrm{dt}$

$\%$ since $\mathrm{v}$ is cantered around centre; six is subtracted from

all velocities

df $(x, y, z, v x, v y, v z)=$

$(v x-c e n t r e) * d f \_d x+(v y-c e n t r e) * d f \_d y+$

$(v z-c e n t r e) * d f \_d z+e \_m *((e x+(v y-c e n t r e) * b z-$

$(\mathrm{vz}-\mathrm{centre}) * \mathrm{by}) * \mathrm{df} \_\mathrm{dvx}+(\mathrm{ey}+(\mathrm{vx}-\mathrm{centre}) * \mathrm{bz}-$

$(\mathrm{vz}-\mathrm{centre}) * \mathrm{bx}) * \mathrm{df} \_\mathrm{dvy}+(\mathrm{ez}+(\mathrm{vx}-\mathrm{centre}) * \mathrm{by}-$

$($ vy-centre)*bx)*df_dvz);

end

end

end

end

end

end

$\mathrm{f}=\mathrm{f}+\mathrm{dt}^{*} \mathrm{df}$;

figure;

subplot $(1,3,1)$; 
imshow(mat2gray(f(1:len,1:len,centre,centre,centre,centre)));

colormap hot;

xlabel ('x');

ylabel ('vx');

title (['PDF at $\mathrm{y}=0, \mathrm{z}=0, \mathrm{vy}=0, \mathrm{vz}=0$ and $\mathrm{t}=$ ' num $2 \mathrm{str}(\mathrm{t})]$ );

subplot $(1,3,2)$;

$\mathrm{g}=\mathrm{f}$ (centre, centre, 1: len, 1:len,centre,centre);

imshow (mat2gray (reshape(g, [len len])));

colormap hot;

Xlabel ('y');

Ylabel ('vy');

Title (['PDF at $\mathrm{x}=0, \mathrm{z}=0, \mathrm{vx}=0, \mathrm{vz}=0$ and $\mathrm{t}=$ ' num2str $(\mathrm{t})]$ );

Subplot $(1,3,3)$;

$\mathrm{g}=\mathrm{f}$ (centre, centre, centre, centre, 1: len, 1: len);

Imshow (mat2gray (reshape (g, [len len])));

Colormap hot;

Xlabel ('z');

Ylabel ('vz');

Title (['PDF at $\mathrm{x}=0, \mathrm{y}=0, \mathrm{vx}=0, \mathrm{vy}=0$ and $\mathrm{t}=$ ' num2str $(\mathrm{t})])$; end

Toc;

\section{MULTI SPECIES COLLISIONS}

According to hydrodynamic theory of multi species diffusion in a gas is governed by given below equation (10) This problem can be worked for taking collision of similar charges, different charges and opposite charges for computing collision integral of multi species .E/N ratio can be evaluated to predict the outcome of collision for multi species. Here we have modeled for multi species collision integral which will give much better insight in understanding of transport physics of gases, here we assume collision of two species at any time because collision of three species is negligible also as per authors knowledge no solutions in this regards have been evaluated for simulations of multi species collision term. The collision integral [1-4] which is necessary for computing the transport properties has been worked upon. Collision integral can be written as sum of contributions of particles interactions with long range attractive portion of intermolecular potential defined .Here we have modeled for multi species collision integral which will give much better insight in understanding of transport physics of gases. Here we assume collision of two species at any time because collision of three species is negligible. Also as per authors knowledge no such solution in this regards have been evaluated for simulations of multi species collision term. Flick diffusion is an approximate method for determining multispecies fluid dynamics .we can compare our model, for multispecies with Fick diffusion. Diffusion process SNR can be evaluated by Brownian motion. After few collisions noise starts to creep and contaminates the results. Noise growth is caused when particles frequency tends to infinity as well as to zero. Molecules process is determined by evaluating cross section evaluation. In our approach we have evaluated collision integral taking two different charge particles and only binary collisions are considered.The approach can be extended for computing collision integral taking different particles into account and summed to evaluate resultant multi species collision integral. For the sake of simplicity no secondary electro emission from the walls is considered. Various parameters i.e. time, ion current, pressure, temperature, number of particles, field, energy distribution function, current, mean free path, $\mathrm{E} / \mathrm{N}$ ratio, collision cross section of ion and gas molecules can be worked. Solving Boltzmann transport equation governing drift and diffusion of the particles rate of the arrival, time, spectrum etc can be predicted .Ionic flux density is given by Flick's law. Study of collision between electron .Collision kernels can be useful tool for carrying simulations. Relaxation time approximation (RTA) is simplification to BTE Integral .We can determine friction and diffusion coefficients that that are due to collisions. Here friction term represents drag or slowdown of particles due to collision and diffusion term producing a spreading of distribution function.

$$
\begin{array}{cll}
\mathrm{f}_{\mathrm{i}}(\mathrm{t}, \vec{r}, \vec{v}) & \text {, where } & \mathrm{i}=1,2,3,4 \ldots \ldots \ldots \ldots \mathrm{N} \\
\mathrm{m}_{\mathrm{i}}= & \text { Mass } \\
\mathrm{e}_{\mathrm{i}}= & \text { Charge }
\end{array}
$$

Distribution function and derivation of collision integral of multi species taking non-reactive pairs into consideration has been worked and can be written as follows:

$$
\begin{aligned}
& \frac{\partial f i}{d x}+\left(v, \nabla_{r}\right) f_{i}+\frac{e i}{m i}(E+\overrightarrow{V B}) \nabla_{v} \cdot f_{i}= \\
& \sum_{j=1}^{N} I_{i j}
\end{aligned}
$$

i species colliding with $\mathrm{j}$ species and evaluating collision integral as below :

$$
\begin{aligned}
& \mathrm{I} \mathrm{ij}= \\
& \sum_{\mathrm{j}} \int\left(-\mathrm{f}_{\mathrm{i}}(\mathrm{t}, \overrightarrow{\mathbf{r}}, \overrightarrow{\mathbf{v}}) \cdot \mathrm{f}_{\mathrm{j}}(\mathrm{t}, \overrightarrow{\mathbf{r}}, \overrightarrow{\mathbf{v}})+\mathrm{f}_{\mathrm{i}}\left(\mathrm{t}, \overrightarrow{\mathbf{r}}^{\mathbf{r}}, \overrightarrow{\mathbf{v}}^{\prime}\right) . \mathrm{f}_{\mathrm{j}}\left(\mathrm{t}, \overrightarrow{\mathbf{r}}^{\prime} \mathbf{i}, \mathrm{v}_{\mathrm{i}}\right) .\right. \\
& \boldsymbol{\sigma}_{\mathrm{ij}}\left(\mathrm{v}, \mathrm{v}_{\mathrm{i}}, \mathrm{v}^{\prime}, \mathrm{v}_{\mathrm{i}}{ }_{\mathrm{i}}\right) \boldsymbol{\delta}^{3} \mathrm{v}_{\mathrm{i}}
\end{aligned}
$$

\section{Coupled EM Transport}

Flow of EM transport through plasma can be modelled as follows:

$$
\begin{aligned}
& \frac{\partial \mathrm{fi}}{\mathrm{dx}}+\left(\mathbf{v}, \nabla_{\mathbf{r}}\right) \mathbf{f}+\frac{\mathrm{ei}}{\mathrm{mi}}(\mathbf{E}+\overrightarrow{\mathrm{VB}}) \nabla_{\mathrm{v}} . \mathbf{f} \\
& =-\frac{\mathbf{f}-\mathbf{f c}}{\tau}
\end{aligned}
$$

Equation (9) presents multi species domain and equation (10) gives coupled concepts. Electromagnetic field E,B has been coupled with $f(x, v, t)$ through Maxwell's system. As the upper part of the atmosphere consists shells of electrons and electrically charged atoms and molecules that surround earth from $50 \mathrm{~K}$ ms to $1000 \mathrm{kms}$. Plasma contain ionized layer known ionosphere. Positive and negative ions are attracted because of EM force Positive and negative ions are attracted because of EM Force We can derive many other parameters like current density, flux, collision integral etc. Collision integral provides us rate of change of PDF. Coupled system can provide us solution without making approximations. Collision integral can 
provide us contribution from particles iterations with long range communication. Power spectrum gives electron density measurement, ion and electron temp, mass, drift velocity. Flux is measurement of intensity of charge. Plasma is a ionized gas . It may responds strongly to EM Field. Aplasma is a collection of particles some positive charged and some negatively charged particles and few neutral. Locally a charge imbalance may exist, which may lead to net electric field in that reason. State of plasma is characterized by distribution function $\mathrm{f}(\mathrm{r}, \mathrm{v}, \mathrm{t})$. Here we have simulated the above equation (14) by recursive method. We have solved for E \& B. For solving these values we have assumed initial function as Gaussian and then evaluated V \& A. For solution of A and V values first we need to compute the values of $\mathrm{J}$ (current density) and $\rho$ (charge density). After computing all the above values we need to substitute all values obtained thus into the above mentioned master equation or coupled equation for solution by iterative method. For computing coupled solution we can also assume Fermi Dirac/ Gaussian as initial function.

Results of simulations are presented in fig 1,2,3 which gives us total insight of precision solution of Boltzmann Transport Equation. This type of solution approach have been unique of its nature so far as compare to previous one.

\section{$\vec{E}(\overrightarrow{\mathbf{r}}, \mathbf{t})=\overrightarrow{-\nabla} \mathbf{v}(\overrightarrow{\mathbf{r}}, \mathbf{t})-\frac{\mathrm{d}}{\mathrm{dt}} \overrightarrow{\mathbf{A}}(\overrightarrow{\mathbf{r}}, \mathbf{t})$ \\ $\vec{B}(\overrightarrow{\mathbf{r}}, \mathbf{t})=\overrightarrow{\nabla^{*}} \times \overrightarrow{\mathbf{A}^{*}}(\overrightarrow{\mathbf{r}}, \mathbf{t})$}

Her E and B are electric and magnetic fields 3D simulation results have been presented in fig 3 .

$\mathbf{V}(\overrightarrow{\mathbf{r}}, \mathbf{t})=\frac{1}{4 \pi \in 0} \int \frac{\mathrm{e}\left(\overrightarrow{\mathbf{r}}^{r}-\mathbf{t}-\frac{\| \vec{r}_{a} \vec{r}^{\prime}}{2}\right)}{\left\|\overrightarrow{\mathbf{r}}_{0} \overrightarrow{\mathbf{r}}^{\prime}\right\|} \mathbf{d}^{3} \mathbf{r}^{r}$

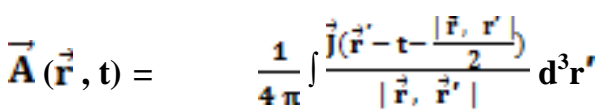

Here $\mathrm{A}$ and $\mathrm{V}$ are electric and magnetic potentials Respectively simulation results are presented

$\begin{array}{lll}\rho(\vec{r}, \mathrm{t}) & = & -\mathrm{e} \int f(\vec{r}, \vec{v}, \mathrm{t}) \mathrm{d}^{3} \mathbf{v} \\ \vec{I}(\overrightarrow{\boldsymbol{r}}, \mathrm{t}) & = & -\mathrm{e} \int \vec{V}(\vec{r}, \vec{v}, \mathrm{t}) \mathrm{d}^{3} \mathbf{v}\end{array}$

Here $\rho$ and $J$ are charge density and current density and simulation results have depicted in fig 16,17 and fig 18,19 respectively. We shall evaluate the value of coupled solution.

$$
\begin{aligned}
& \frac{\partial f(\vec{r}, \mathrm{t})}{\partial t}+(\vec{V}, \vec{\nabla}) \mathbf{f} f(\vec{r}, \vec{V}) \\
& -\frac{e}{m}\left(\vec{E}+\vec{V} \times \vec{B}, \nabla_{\mathrm{V}}\right) \\
& \mathbf{f}(\overrightarrow{\boldsymbol{r}}, \vec{v}, \mathrm{t}) \quad=\quad \frac{\mathrm{fo}-\mathrm{f}}{\tau} \\
& {\left[\frac{\partial f}{\partial t}\right]_{\mathrm{coll}}=\int \boldsymbol{\sigma}(\mathrm{v},)\left|\boldsymbol{V}-\boldsymbol{V}^{\prime}\right|(-\mathbf{f}(\overrightarrow{\boldsymbol{r}}, \vec{v}, \mathrm{t})+} \\
& \mathrm{f}\left(\overrightarrow{\boldsymbol{r}}, \vec{v}^{\prime}, \mathrm{t}\right)+\mathrm{f}(\overrightarrow{\boldsymbol{r}}, \vec{v}, \mathrm{t}) \mathrm{f}\left(\overrightarrow{\boldsymbol{r}}, \overrightarrow{v^{\prime}}, \mathrm{t}\right) \mathrm{d}^{3} \mathbf{V}^{\mathrm{t}} \operatorname{Sin} \theta \mathrm{d} \theta
\end{aligned}
$$

Taking initial function as $\mathrm{f}_{0}(\vec{r}, \vec{v}, \mathrm{t})$ as Gaussian function. We have evaluated value of $\mathrm{E}, \mathrm{B}, \mathrm{A}, \mathrm{V}, \mathrm{J}$ and $\rho$. These values have been substituted in the coupled equation and simulated results are obtained for coupled equation.

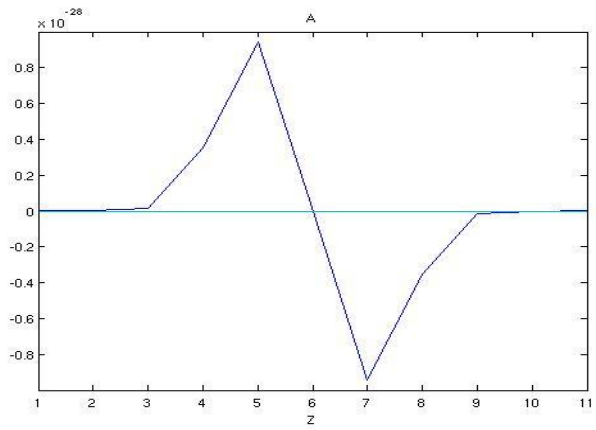

Fig.3(a) A vs $\mathrm{Z}$ at specific value $\mathrm{x}, \mathrm{y}, \mathrm{t}$ mid points

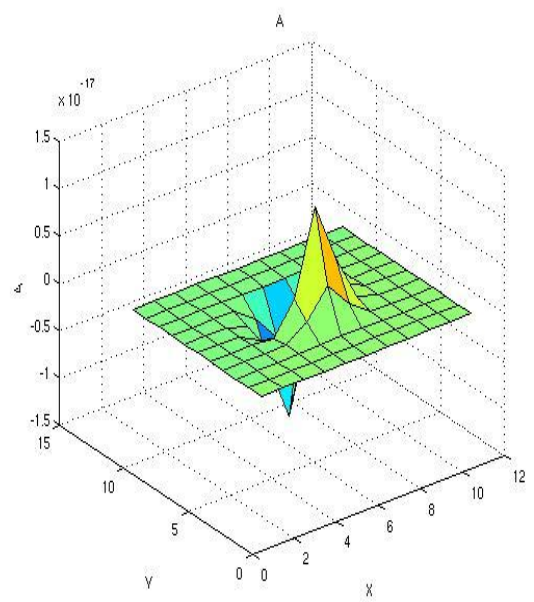

Fig3(b) A vs $\mathrm{X}, \mathrm{Y}$ at specific value $\mathrm{z}, \mathrm{t}$ mid points

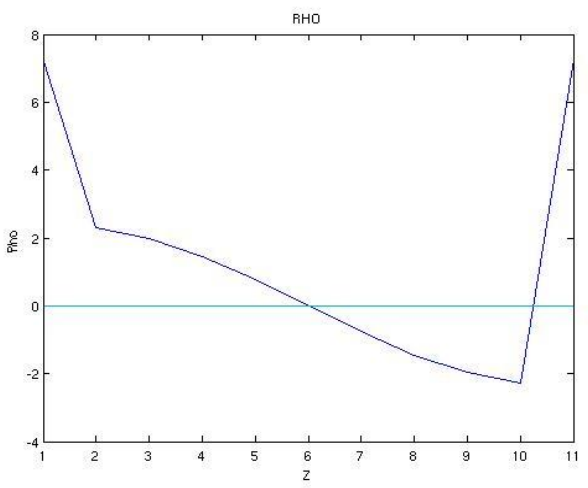

Fig.3(c) $\quad \rho(\vec{r}, \mathrm{t})$ charge density rho vs. $\mathrm{Z}$ at specific mid points $\mathrm{x}, \mathrm{y}, \mathrm{t}$.

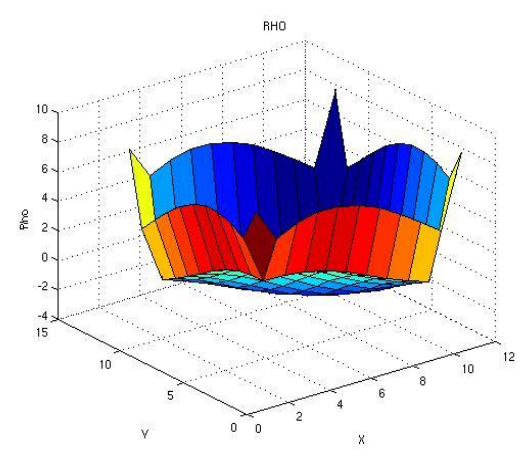


Fig . 3(d) Rho vs $\mathrm{X}, \mathrm{Y}$ at specific value $\mathrm{z}, \mathrm{t}$ mid points

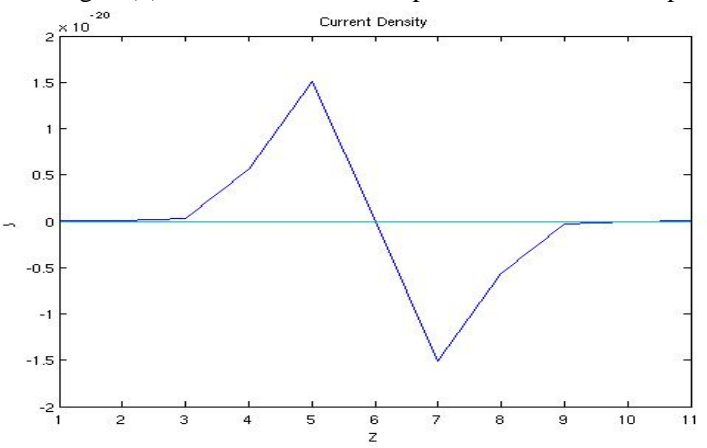

Fig .3(e) $\mathrm{J}$ at specific value $\mathrm{x}, \mathrm{y}, \mathrm{t}$ mid points w.r.t to $\mathrm{Z}$.

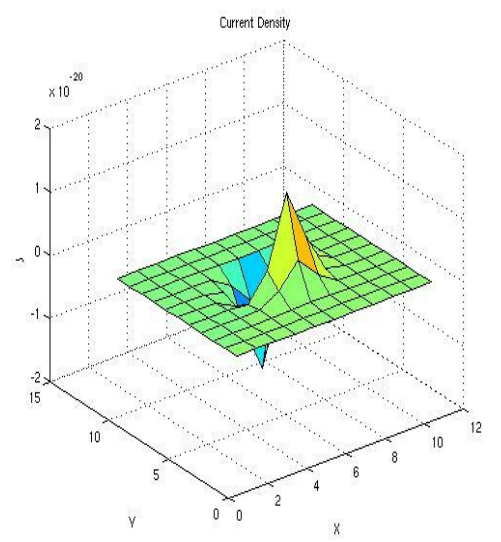

Fig.3(f) J vs $\mathrm{X}, \mathrm{Y}$ at specific value $\mathrm{z}, \mathrm{t}$ mid points .

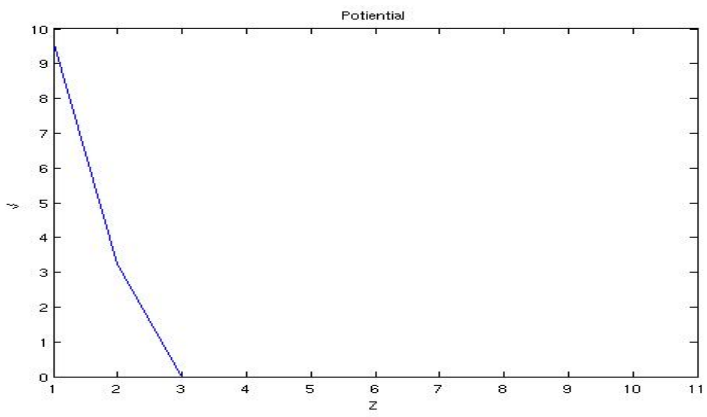

Fig( 3g) V vs $Z$ at specific value $x, y, t$ mid points

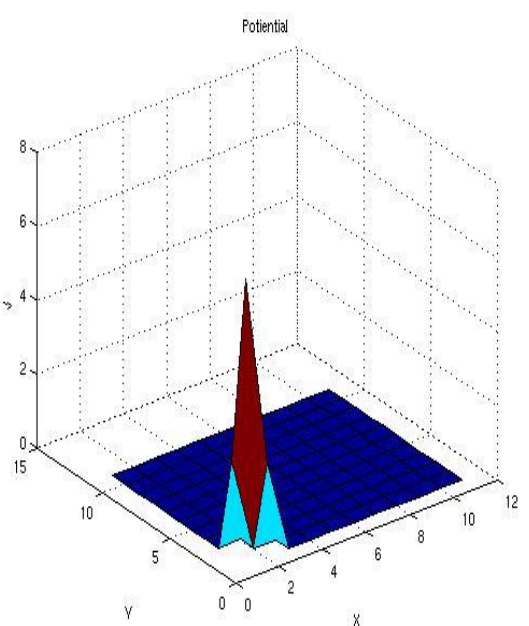

Fig .3(h) V vs $\mathrm{X}, \mathrm{Y}$ at specific value $\mathrm{z}, \mathrm{t}$ mid points

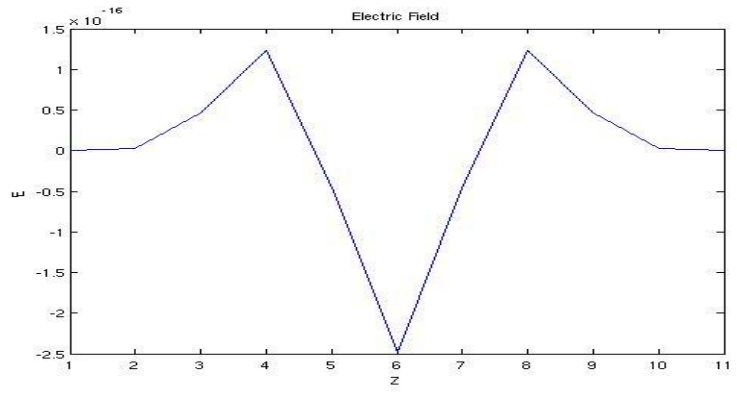

Fig .3(i) E vs $\mathrm{Z}$ at specific value $\mathrm{x}, \mathrm{y}, \mathrm{z}$ mid points

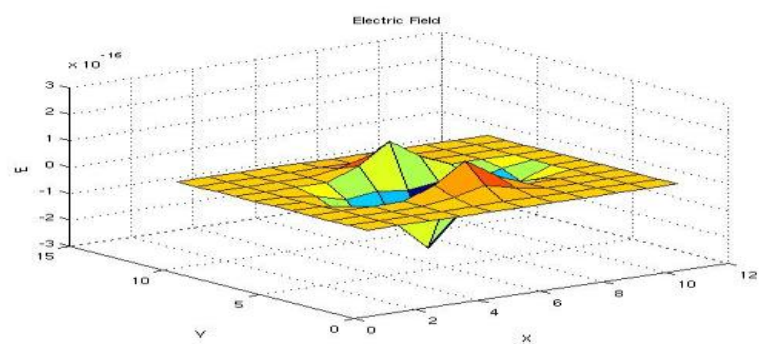

Fig.3(j) E vs $X, Y$ at specific value z,t mid points

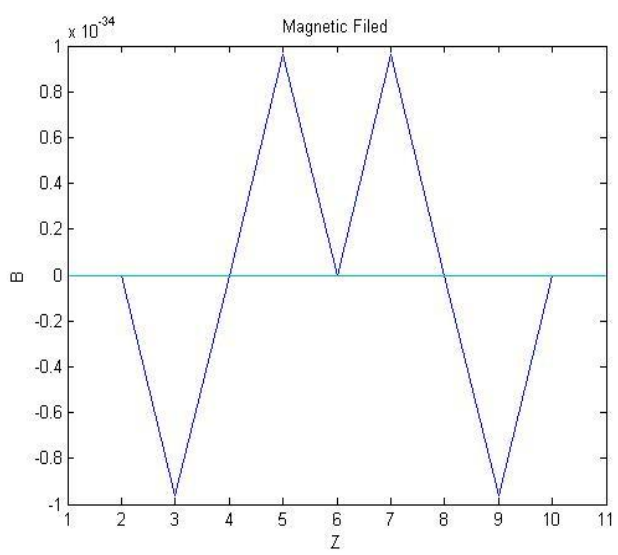

Fig 3(k) B vs $\mathrm{Z}$ at specific value $\mathrm{x}, \mathrm{y}, \mathrm{t}$ mid points 


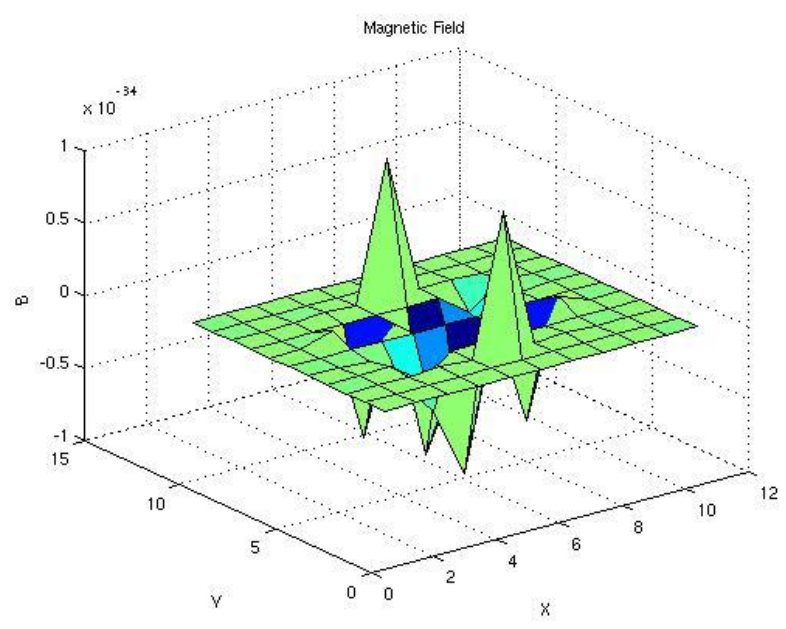

Fig3( 1) B vs X,Y at specific value $z, t$ mid points

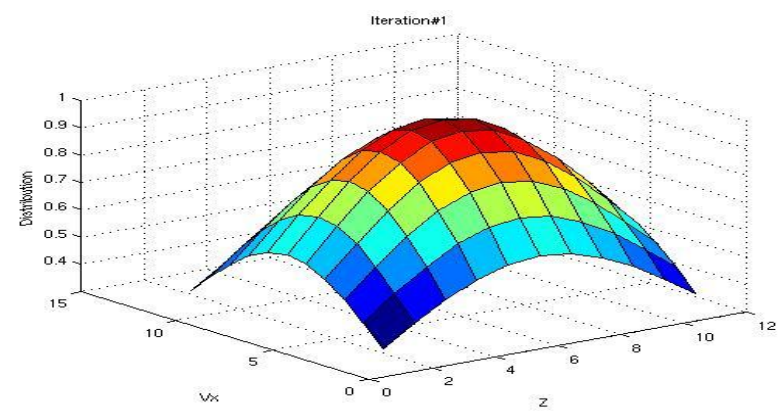

Fig.3(m) PDF vs Vx, Z.

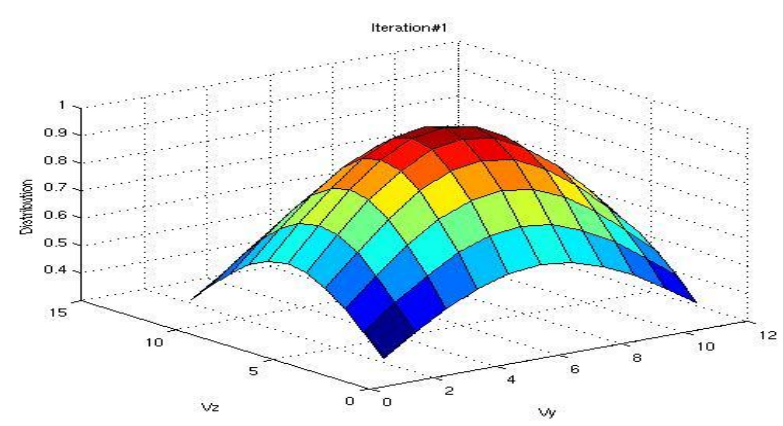

Fig.3(n) PDF vs Vy, Vz

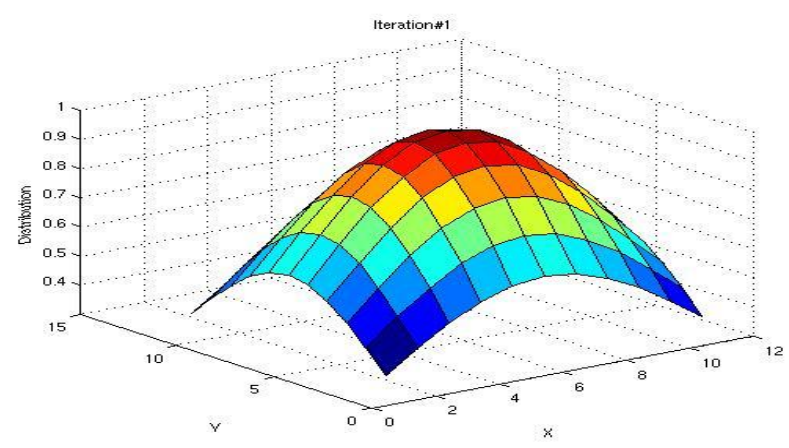

Fig.3(o) PDF vs Vy, Vz

\section{CONCLUSION}

Vol. 1, No. 6, December 2010

Investigations for transport parameters have been worked by means of modeling of Boltzmann transport equation for both linear and nonlinear responses. Collision Integral solution for binary and multi species have been realized and simulated. General algorithms for 3D transport parameters solution have been proposed. 2D transport with and without field have been worked. Multi species terms have been realized. In addition we have proposed an efficient solution to coupled BoltzmannMaxwell's equations.

Convergence solution for 2D model have been evaluated. Coupled results seems to feature pulse nature and presented in results of coupled solutions. This feature with controlled pulse can be used for fast switching.

Modeling of coupled process is difficult because of involvement of two different physics which require time and space analysis. Parameters that control the shape and amplitude of the pulse have been modeled and simulated.

Mismatch of functions having various size of matrix need critical computations. Different dimensions due interdependency of many parameters need to be resolved during code development for 3D analysis in iterative simulation. Application of our work can be in chip designing in microelectronics, Plasma antenna designs and study of upper atmosphere characteristics.

\section{ACKNOWLEDGEMENT}

I am very thankful to Prof Raj Senani, Director and Prof Asok De, Director for providing all required resources for this research Work. I am indebted to my wife Sujata, daughter Monica and my son Rahul for giving me research environment.

\section{REFERENCES}

[1] Statistics Mechanics, Kerson Huang, Wiley 2008

[2] Bahadir, A.R .and T. Abbasov (2005) "Numerical investigation of the liquid flow velocity over an infinity plate which is taking place in a magnetic field" International journal of applied electromagnetic and mechanics.

[3] EM Lifshitz and LD Landau Electrodynamics of continuous media"butterworth-Hienemann

[4] Fermigier, M. (1999), "Hydrodynamique physique" Problèmes résolus avec rappels de cours, collectionsciences sup Physique, edition Dunod.

[5] Wait, R. (1979) "The numerical solution of algebraic equations" A Wileyinterscience publication.

[6] Chorin, A.J., 1986, "Numerical solution of the Navier-Stokes equations", Math. Comp., Vol. 22, pp. 745-762.

[7] EM Lifshitz and LD Landau "Fluid Mechanics" Vol 6, ButterworthHeinemann.

[8] Holman, J.P., 1990, "Heat Transfer" ( $7^{\text {th }}$ edition), Chapter 12, Special Topics in Heat Transfer, MacGraw-Hill Publishing Company, New York.

[9] Guermond, J.L. \& Shen, J., 2003, "Velocity-correction projection methods for incompressible flows", SIAM J. Nume. Anal. Vol. 41, No. 1, pp. 112-134.

[10] Ramesh Garg "Analytical and Computational methods in electromagnetic" Artech House, London

[11] JD Jackson, "Classical Electrodynamics" third edition, Wiley.

\section{AUTHOR's PROFILE}


Rajveer S Yaduvanshi has been working as Asst Professor in ECE Vol. 1, No. 6, December 2010

department of AIT, Government of Delhi, Delhi -110031. He has 21

years of teaching experience. He is Fellow of IETE. Author has visited

France for radar moderanization program representing India. His research interest has been device physics and satellite communication. He has published six research papers in intenational journals and conferences.

Professor Harish Parthasarathy has been working as full professor in ECE depertment of NSIT Dwarka, Delhi-110075. His area of research is DSP. $\mathrm{He}$ has published several books in this domain and has guided five $\mathrm{PhD}$ students and guiding our more $\mathrm{PhD}$ students under Delhi University. 